\title{
ENFRENTAMENTO DA VIOLÊNCIA SEXUAL INFANTO-JUVENIL NA PERSPECTIVA DOS PARTICIPANTES DE UM CURSO DE FORMAÇÃO
}

\author{
Verônica Borges Kappel ${ }^{1}$, Daniela Tavares Gontijo ${ }^{2}$, Helena Hemiko Iwamoto ${ }^{3}$, Rogéria Moreira Rezende Isobe ${ }^{4}$
}

\begin{abstract}
RESUMO: A violência sexual infanto-juvenil é um fenômeno demarcado pela multiplicidade de fatores cujo enfrentamento requer participação de diferentes atores/setores sociais. Esta pesquisa, descritiva e exploratória, objetivou identificar as dificuldades, pessoas e/ou instituições envolvidas e estratégias de enfrentamento da violência na perspectiva de 57 participantes do Curso de Formação do Programa de Ações Integradas e Referenciais de Enfrentamento à Violência Sexual Infanto-juvenil. Os dados foram coletados por meio de questionário, analisados por análise de conteúdo e descritos por frequência de aparição. Aspectos culturais, familiares e políticos constituíram as principais dificuldades encontradas; a rede de atendimento apareceu com maior frequência como responsável pelo enfrentamento envolvendo ações preventivas à violência infanto-juvenil e ao fortalecimento da rede de proteção. Os dados corroboraram a necessidade de propostas e estratégias de enfrentamento do fenômeno que abordem a formação dos profissionais que compõem a rede de proteção de crianças/adolescentes.

PALAVRAS-CHAVE: Violência sexual; Maus-tratos sexuais infantis; Defesa da criança e do adolescente.

\section{SEXUAL VIOLENCE OF CHILDREN AND TEENAGERS CONFRONTATION FROM THE PERSPECTIVE OF PARTICIPANTS IN THE TRAINING COURSE}

\begin{abstract}
Child and youth sexual violence is a phenomenon characterized by the multiplicity of factors whose confronting requires the participation of different actors and social sectors. This descriptive and exploratory research aimed to identify the difficulties, people and/or institutions involved and the strategies for dealing with violence from the perspective of 57 participants in the training course for the Integrated Actions Referring to Confronting Child and Youth Sexual Violence Program. The data was collected by means of a questionnaire, was analyzed by content analysis and described by frequency of apparition. Cultural, family and political aspects were the principal difficulties met; the care system appeared with the highest frequency as responsible for confrontation involving actions aimed at preventing child-youth violence and strengthening of the protection network. The data supported the need for proposals and strategies for confronting the phenomenon which affect the training of the professionals who make up the network for the protection of children and adolescents.
\end{abstract}

KEYWORDS: Sexual violence; Sexual maltreatment of children; Defence of the child and adolescent.

\section{LA PERSPECTIVA DE LOS PARTICIPANTES DEL CURSO DE CAPACITACIÓN SOBRE EL ENFRENTAMIENTO DE LA VIOLENCIA INFANTO-JUVENIL}

RESUMEN: La violencia sexual infantojuvenil es un fenómeno demarcado por la multiplicidad de factores cuyo afrontamiento necesita la participación de diferentes actores/sectores sociales. Esta investigación, descriptiva y exploratoria, tuvo el objetivo de identificar las dificultades, personas y/o instituciones involucradas y estrategias de afrontamiento de la violencia en la perspectiva de 57 participantes del Curso de Formación del Programa de Acciones Integradas y Referenciales de Afrontamiento a la Violencia Sexual Infantojuvenil. Los datos fueron recogidos por medio de cuestionario, analizados por análisis de contenido y descriptos por frecuencia en que aparecieron. Aspectos culturales, familiares y políticos constituyeron las principales dificultades encontradas; la red de atendimiento apareció con mayor frecuencia como responsable por el afrontamiento involucrando acciones preventivas a la violencia infantojuvenil y al fortalecimiento de la red de protección. Los datos corroboraron la necesidad de propuestas y estrategias de afrontamiento del fenómeno sobre la formación de los profesionales que componen la red de protección de niños/adolescentes. PALABRAS CLAVE: Violencia sexual; Malos tratos sexuales infantiles; Defensa del niño y del adolescente.

\footnotetext{
${ }^{1}$ Terapeuta Ocupacional. Mestranda pelo Programa de Pós-Graduação em Atenção à Saúde da Universidade Federal do Triângulo Mineiro - UFTM.

${ }^{2}$ Terapeuta Ocupacional. Doutora em Ciências da Saúde. Professora do Curso de Graduação em Terapia Ocupacional da Universidade Federal de Pernambuco - UFPE e do Programa de Pós-Graduação em Atenção à Saúde da UFTM.

${ }^{3}$ Enfermeira. Doutora em Enfermagem Fundamental. Professora do Curso de Graduação em Enfermagem e do Programa de PósGraduação em Atenção à Saúde da UFTM.

${ }^{4}$ Pedagoga. Doutora em Educação. Professora do Departamento de Educação da UFTM.
}

Autor correspondente:

Recebido: 21/09/2011

Daniela Tavares Gontijo Aprovado: 10/06/2012

Universidade Federal de Pernambuco

Rua Prof. Moraes Rego, s/n - 50740-600 -Recife-PE-Brasil

E-mail: danielatgontijo@gmail.com 


\section{INTRODUÇÃO}

A violência contra crianças e adolescentes, historicamente, acompanha a trajetória humana das relações sociais, familiares, institucionais e encontra-se presente em variados contextos, sendo caracterizada, especialmente, pela violência intrafamiliar e doméstica ${ }^{(1-2)}$. No que se refere as suas modalidades, a violência pode ser compreendida como negligência/abandono, violência física, violência psicológica e violência sexual ${ }^{(3)}$.

A violência sexual contra crianças e adolescentes, também denominada violência sexual infanto-juvenil (VSIJ) é considerada um problema de saúde pública pela Organização Mundial da Saúde, sendo uma realidade frequente em muitas partes do mundo. Estima-se que em 2002, 150 milhões de meninas e 73 milhões de meninos foram vitimizados pela $\mathrm{VSIJ}^{(4)}$.

De uma forma geral, a VSIJ constitui toda e qualquer situação em que a criança ou o adolescente é usado para a satisfação sexual de pessoas mais velhas, sendo que as relações assimétricas de poder entre a vítima e o abusador são as que mais identificam esta situação ${ }^{(5-6)}$. A VSIJ engloba situações com e sem contato físico, podendo se caracterizar como abuso sexual intrafamiliar, abuso sexual extrafamiliar e exploração comercial sexual de crianças e adolescentes ${ }^{(5)}$.

No que se refere às causas da VSIJ, compreende-se que esta se caracteriza como um fenômeno complexo fruto das inter-relações de fatores individuais, culturais, sociais, políticos e econômicos ${ }^{(6)}$. Entre suas consequências para o desenvolvimento das crianças e adolescentes, destacam-se alterações físicas decorrentes de lesões e contaminações por doenças sexualmente transmissíveis, alterações psicológicas e emocionais, mudanças de hábitos e rotinas, dificuldades no estabelecimento de relações afetivas e amorosas, assim como na vida sexual, engajamento em trabalhos sexuais (prostituição) e dependência de substâncias psicoativas, licitas e ilícitas ${ }^{(3,7)}$.

Considerando todos estes aspectos, compreende-se a importância do enfrentamento da VSIJ, que se caracteriza como uma violação dos direitos das crianças e adolescentes. No Brasil, este enfrentamento é direcionado pelo Plano Nacional de Enfrentamento da Violência Sexual InfantoJuvenil, publicado em 2002 pela então Secretaria de Estado dos Direitos Humanos, vinculada ao Ministério da Justiça. Este Plano é um instrumento de garantia e defesa de direitos de crianças e adolescentes que pretende criar, fortalecer e implementar um conjunto articulado de ações e metas fundamentais para assegurar a proteção integral à criança $\mathrm{e}$ ao adolescente em situação ou risco de violência sexual ${ }^{(8)}$.
O Plano Nacional de Enfrentamento da Violência Sexual Infanto-Juvenil é operacionalizado nos municípios por meio do Programa de Ações Integradas e Referenciais de Enfrentamento da Violência Sexual Infanto-Juvenil no Território Brasileiro (PAIR) ${ }^{(9)}$. Este Programa tem abrangência nacional, em expansão, e está presente em 474 cidades brasileiras de 24 unidades federativas. O PAIR, que tem como principio básico a criação e o fortalecimento de redes locais de enfrentamento da VSIJ, é sistematizado em ações que envolvem a Articulação Político-Institucional; o Diagnóstico Rápido Participativo; o Seminário para Construção do Plano Operativo Local; a Assessoria Técnica e o Monitoramento dos Planos Operativos Locais e Capacitação da Rede ${ }^{(9)}$.

A Capacitação da Rede, sistematizada em cursos de formação e oficinas, é compreendida como uma das estratégias de maior impacto no PAIR que envolve todos os segmentos da rede de proteção e sistemas de garantias no município. Engloba a participação de profissionais da saúde, assistência social, educação, defesa e responsabilização, transporte, mídia e turismo, entre outros ${ }^{(9)}$.

Considerando estes aspectos, esta pesquisa teve como objetivo identificar as dificuldades, pessoas/instituições envolvidas e as estratégias de enfrentamento da VSIJ na perspectiva dos participantes do curso de formação do PAIR no Triângulo Mineiro.

\section{MÉTODO}

Pesquisa exploratória e descritiva realizada com 57 profissionais de 26 municípios, que participavam do curso de formação do projeto "Minas Gerais no enfrentamento à violência sexual contra crianças e adolescentes - expansão do PAIR para a região do Triângulo Mineiro", em dezembro de 2010, na cidade de Uberaba-MG.

Destaca-se que a escolha dos profissionais participantes no curso de formação foi de responsabilidade de cada município, que indicaram os sujeitos que deveriam conduzir o processo do PAIR em seus municípios. Os critérios de inclusão dos sujeitos foi ser participante do referido curso de formação. Após seu assentimento formal, a coleta de dados foi realizada no primeiro dia do curso, em momento anterior às atividades programadas, considerando ser importante a identificação das concepções iniciais dos sujeitos e que o curso poderia influenciar nestas. Os dados foram coletados por meio de um questionário autoaplicável, elaborado pelas pesquisadoras, que continha questões fechadas, 
relacionadas aos dados sociodemográficos, e questões abertas, referentes à percepção dos sujeitos em relação às dificuldades para o enfrentamento do fenômeno, identificação das pessoas e/ou instituições responsáveis por este processo, assim como as possíveis estratégias para lidar com o problema em pauta.

A análise dos dados se deu por meio da análise de conteúdo temática ${ }^{(10)}$. As três categorias temáticas, resultantes do processo de análise e que se relacionam com o conteúdo do instrumento e com o referencial teórico adotado, foram descritas em termos de frequência absoluta de aparição. Considerando que em uma mesma resposta à questão aberta foi possível identificar diferentes unidades temáticas, neste estudo não foi possível a utilização de medidas de frequência relativa.

O projeto de pesquisa foi aprovado pelo Comitê de Ética da Universidade Federal do Triângulo Mineiro (UFTM) de acordo com o protocolo n. 1589.

\section{RESULTADOS}

Participaram da primeira etapa do curso de formação do PAIR/Minas e da pesquisa 57 profissionais de 26 municípios, sendo a maioria mulheres $(94,73 \%)$, com pós-graduação lato-sensu (47,36\%), idade média de 34,87 anos. A maioria dos participantes atuava no campo da assistência social (18), seguidos dos profissionais da área da saúde (14), agentes que atuam em cargos de gestão (13), área da educação (5), campo administrativo (5) e conselheiros tutelares (2).

Na primeira categoria, Dificuldades encontradas no enfrentamento da VSIJ, os participantes apontaram aspectos culturais, familiares e questões vinculadas às características das políticas e serviços (Tabela 1).

No que se refere aos aspectos culturais, foi referida a "cultura do silêncio", a qual pode refletir no baixo índice de denúncias e foi identificada como a principal barreira no enfrentamento do fenômeno da violência. O limitado comprometimento dos familiares na assistência às crianças/adolescentes foi a segunda maior dificuldade identificada.

Os sujeitos do PAIR/Minas pontuaram também como dificuldade a escassez de políticas públicas, especialmente a fragilidade das medidas punitivas e $o$ despreparo dos profissionais para lidar com a questão da VSIJ. Foi destacado o despreparo e o pouco conhecimento sobre a legislação vigente relacionada a esta questão. As limitações no processo de mobilização e do trabalho em rede, e o pouco comprometimento dos órgãos competentes para o enfrentamento da VSIJ também foram destacados pelos sujeitos. Ressalta-se que somente um sujeito indicou aspectos relacionados à desigualdade social e pobreza como um obstáculo para o enfrentamento da VSIJ, sendo importante considerar que cinco sujeitos responderam a questão de forma vaga e inespecífica, apontando que as dificuldades são grandes e que sempre perdurarão.

$\mathrm{Na}$ segunda categoria de análise, Pessoas elou Instituições Responsáveis pelo Enfrentamento da $V S I J$, instituições e profissionais vinculados à rede de atendimento às crianças e adolescentes foram os mais citados, especialmente aqueles relacionados à educação, saúde e assistência social (Tabela 2).

Tabela 2 - Pessoas e/ou instituições identificadas como responsáveis pelo enfrentamento da violência sexual infanto-juvenil pelos participantes do curso de formação do PAIR/Minas. Uberaba, 2010

\begin{tabular}{lc}
\hline Pessoas- Instituições & F \\
\hline Pertencentes à rede de atendimento & 213 \\
Pertencentes à rede de defesa e dos direitos & 31 \\
Governos municipais, estaduais e federal & 27 \\
Pertencentes à rede de responsabilização & 25 \\
\hline
\end{tabular}

Tabela 1 - Dificuldades relatadas pelos participantes do curso de formação do PAIR/Minas no enfrentamento da violência sexual infanto-juvenil. Uberaba, 2010

\begin{tabular}{lc}
\hline DIFICULDADES & F \\
\hline Relacionadas aos aspectos culturais & 20 \\
Pouco comprometimento das famílias & 18 \\
Escassez de políticas públicas voltadas para o enfrentamento da violência sexual infanto-juvenil & 13 \\
Falta de preparo profissional para lidar com a temática & 13 \\
Fragilidade do trabalho em rede & 08 \\
Pouco comprometimento dos órgãos competentes & 07 \\
Resposta inespecífica & 05 \\
Pouco comprometimento dos profissionais & 02 \\
Relacionadas aos aspectos sociais & 01 \\
\hline
\end{tabular}


Além da rede de atendimento, os sujeitos do PAIR/ Minas responsabilizaram as pessoas/instituições pertencentes à rede de defesa dos direitos, como o Conselho Tutelar e o Conselho Municipal dos Direitos da Criança e do Adolescente pelo enfrentamento da VSIJ.

Não houve citação por parte dos participantes, quando comparadas com a rede de atendimento e a rede de defesa dos direitos, das instâncias governamentais e da rede de responsabilização, por exemplo da Segurança Pública e do Ministério Público, como atores envolvidos no enfrentamento da VSIJ.

$\mathrm{Na}$ terceira categoria temática, Estratégias de enfrentamento da VSIJ, os participantes apontaram as ações preventivas como a principal forma de enfrentamento do fenômeno, seguido do fortalecimento da rede de proteção (Tabela 3).

Tabela 3 - Estratégias de enfrentamento da violência sexual infanto-juvenil relatadas pelos participantes do curso de formação do PAIR/Minas. Uberaba, 2010

\begin{tabular}{lc}
\hline Estratégias de enfrentamento & F \\
\hline Ações preventivas & 47 \\
Fortalecimento da rede de proteção & 37 \\
Implantação de políticas públicas voltadas para & 14 \\
a problemática & 08 \\
Resposta inespecífica & \\
\hline
\end{tabular}

No que diz respeito às ações preventivas relacionadas ao enfrentamento da VSIJ, as principais ações mencionadas pelos sujeitos do PAIR/Minas foram palestras, debates, campanhas educativas, divulgação da temática na mídia, escola e comunidade em geral, bem como atuação com as famílias dos casos notificados para prevenir a repetição do ciclo da vitimização.

O fortalecimento da rede de proteção, na perspectiva dos participantes está vinculado, principalmente, à melhor capacitação dos profissionais e à realização do diagnóstico da situação em cada município, o que poderá contribuir para a efetivação de um trabalho integrado e sistêmico. Destaca-se a ocorrência de 8 participantes que não mencionaram, em suas respostas, ações específicas de enfrentamento da VSIJ.

\section{DISCUSSÃO}

As estratégias de enfrentamento da VSIJ trazidas pelos participantes refletem a complexidade que caracteriza o fenômeno em estudo, visto que foi apontada a necessidade, tanto de ações locais - mais pontuais e diretivas - como de ações que envolvem a transformação de concepções cultural e historicamente construídas sobre o fenômeno que impactam na formação e atuação do profissional da rede de proteção.

Quando se analisa as dificuldades para o enfrentamento da VSIJ observa-se uma congruência com outros estudos $^{(11-13)}$ que indicam a subnotificação - validada por uma "cultura do silêncio" em torno da questão da violência sexual - como um dos principais obstáculos encontrados pelos sujeitos participantes.

Embora não explicitada diretamente pelos sujeitos, a subnotificação da VSIJ, por parte de familiares, conhecidos, profissionais e das próprias vítimas, está intrinsecamente relacionada aos aspectos culturais e historicamente construídos, principalmente no que se refere à assimetria de poder presente em diferentes dimensões das relações sociais e que colocam crianças/ adolescentes em situação de opressão ${ }^{(11)}$.

Nesse sentido, significações culturais direcionadas para as crianças e adolescentes estão imersas em concepções adultocêntricas e patriarcais, agravadas por condições de vida marcadas pela segregação e exclusão social, o que consolida cada vez mais o processo de naturalização e banalização da violência.

Considerando este aspecto, na categoria Estratégias de enfrentamento da VSIJ os sujeitos apontaram intervenções com potencial para o aumento das notificações pela sociedade como um todo e explícitas, especialmente, nos temas vinculados às ações preventivas e no fortalecimento da rede de enfrentamento.

A prevenção da ocorrência da VSIJ é um dos eixos norteadores do PAIR ${ }^{(9)}$ e implica tanto em ações que promovam mudanças de concepções culturais sobre $o$ lugar social de crianças e adolescentes, como o fortalecimento das instituições e pessoas responsáveis pela garantia dos direitos infanto-juvenis, também assegurados pela Doutrina da Proteção Integral preconizado pelo Estatuto da Criança e do Adolescente (ECA) ${ }^{(14)}$.

$\mathrm{O}$ fortalecimento das instituições e o aprimoramento profissional dos responsáveis pelo enfrentamento perpassam pela efetivação do trabalho em rede, identificado pelos sujeitos do PAIR/Minas, de forma concomitante, como uma das principais dificuldades no enfrentamento e como potencial estratégia para lidar com a VSIJ.

Assim como referido neste estudo, outros autores apontam que, embora exista ação conjunta e articulada entre algumas instituições no enfrentamento da VSIJ, predomina a falta de conexão e interação da rede de proteção infanto-juvenil como um todo. Isso se deve à escassez de trocas de informações no que diz respeito ao sistema 
de referência e contra-referência e à inexistência de uma definição transparente acerca do papel desempenhado pelos agentes institucionais dentro da rede $\mathrm{e}^{(5,12,15)}$.

A fragilidade da rede de proteção também se reflete na análise dos dados referentes à categoria temática Pessoas elou Instituições Responsáveis pelo Enfrentamento da $V S I J$, na qual se observa uma diferença na frequência de responsabilização quando se compara os diferentes setores que compõem a proteção de crianças e adolescentes; e também na ausência de referência a pessoas ou setores vinculados à sociedade civil, de uma forma geral.

Constata-se a atribuição de maior responsabilização no enfrentamento da VSIJ à rede de atendimento as crianças e adolescentes, aos serviços e programas de proteção especial, e a organizações não governamentais que atuam nesta área ${ }^{(15)}$. A concepção dos participantes pode estar relacionada, por um lado, ao seu perfil profissional, caracterizado por atuar majoritariamente na rede de atendimento, e por outro lado, com uma possível fragilidade e simplificação na concepção do trabalho em rede.

A perspectiva do "trabalho em rede" busca redimensionar as ações de enfrentamento de um fenômeno de tal complexidade e contribuir para a superação de posturas e práticas historicamente construídas e cristalizadas em políticas públicas tradicionais, que se caracterizam pela fragmentação, simplificação, centralização e temporalidade localizada ${ }^{(16)}$. A ação em rede requer, além da mudança na cultura profissional mencionada, a integração das diversas políticas públicas voltadas para a promoção dos direitos da criança e do adolescente; a articulação das ações governamentais e não governamentais na assistência à clientela ${ }^{(17)}$. Ainda, a interação de programas e serviços de atendimento; a identificação de estratégias de acompanhamento e avaliação de políticas e qualidade dos serviços prestados; e a mobilização da sociedade para participar na política do atendimento através do controle social compartilhado e planejado ${ }^{(17)}$.

Nessa perspectiva, o enfrentamento da VSIJ envolve, além da rede de atendimento - composta por instituições executoras de políticas sociais de saúde, educação, assistência social, trabalho, cultura, lazer e profissionalização - a participação da sociedade civil como um todo, da rede de defesa de direitos - composta por órgãos como Conselhos Tutelares, Varas da Infância e da Juventude, Defensoria Pública e Centros de Defesa, os quais têm a função de defender e garantir os direitos de todos os envolvidos na situação de violência sexual notificada - e da rede de respon- sabilização composta pelas Delegacias de Polícia, Delegacias Especializadas, Instituto Médico Legal, Varas Criminais, Vara da Criança e da Adolescência ou da Infância e da Juventude, Delegacia da Criança e do Adolescente e Ministério Público, os quais têm como papel a responsabilização judicial de violações de direitos, proteger a sociedade, e por fim, assegurar o cumprimento da lei ${ }^{(15)}$.

A articulação e potencialização da rede de proteção também requerem, do ponto de vista dos sujeitos deste estudo, um investimento nos programas de capacitação profissional evidenciando que o trabalho isolado como uma dificuldade no enfrentamento da VSIJ. Nessa direção, um dos desafios que se apresentam aos programas de formação profissional continuada consiste em contribuir para a necessária ruptura com uma cultura pautada no isolamento, na fragmentação das ações e na competitividade para a construção de práticas que favoreçam uma intervenção sólida e consistente por meio da reflexão conjunta, do trabalho interdisciplinar, do espírito de cooperação e do planejamento coletivo.

A capacitação da rede de proteção, conforme mencionado anteriormente, se caracteriza como uma das principais estratégias do PAIR, sendo considerada fundamental para ampliação da efetividade das ações de enfrentamento da VSIJ ${ }^{(12)}$. A capacitação deve abordar aspectos teóricos sobre a conceituação, causas e consequências da VSIJ, propiciar a criação de espaços de desenvolvimento e exercício de habilidades necessárias para as intervenções cotidianas, e maior apropriação das políticas públicas e diretrizes governamentais e legais direcionadas para o fenômeno em estudo.

No que se refere às dificuldades de enfrentamento da VSIJ, os dados analisados evidenciam a ênfase atribuída às questões familiares. Entretanto, nota-se que os sujeitos não mencionaram possíveis estratégias de enfrentamento direcionadas especificamente para o fortalecimento da família como fonte de proteção à criança e adolescente. Vale ressaltar que, de acordo com o ECA ${ }^{(14)}$, é dever da família, do Estado e da sociedade a garantia das condições essenciais para o desenvolvimento e do bem estar das crianças e adolescentes.

Embora não tenha sido foco de análise nesta pesquisa, estudos têm apontado um cenário de vulnerabilização crescente das famílias com impacto negativo direto no seu potencial de cuidado em relação às crianças e aos adolescentes ${ }^{(18)}$. Nesse sentido, compreende-se que políticas e ações direcionadas para o fortalecimento da família, nas dimensões econômicas e sociais, são instrumentos importantes 
de enfrentamento à violência de uma forma geral. É importante destacar que a VSIJ caracteriza-se como uma problemática não restrita a estratos econômicos menos privilegiados. No entanto, compreende-se que as situações de pobreza e miséria se constituem como agravantes da situação e limitantes das possibilidades de enfrentamento pelos grupos familiares.

Finalmente, é necessário discutir a percepção dos sujeitos sobre as políticas públicas direcionadas para a VSIJ. Este estudo apontou a necessidade de maior apropriação dos profissionais acerca das políticas e diretrizes governamentais e legais relacionadas ao enfrentamento da VSIJ, assim como da necessidade de discussão e ampliação destas. Nesse sentido, considera-se de suma importância o desenvolvimento de pesquisas que avaliem, sistematicamente e com a utilização de indicadores quantitativos e qualitativos, o impacto do Plano Nacional de Enfrentamento à VSIJ no cotidiano real das crianças e adolescentes brasileiros.

\section{CONSIDERAÇÕES FINAIS}

Este estudo possibilitou a identificação das ações de enfrentamento à VSIJ e pessoas/instituições responsáveis por este processo, assim como as maiores dificuldades na perspectiva dos participantes do curso de formação do PAIR/Minas.

A análise demonstra congruência entre a realidade vivenciada pelos sujeitos no âmbito municipal e a proposta do PAIR/Minas, que estabelece a formação continuada dos profissionais como uma de suas estratégias de ação. Nesse sentido, foi possível identificar a importância dos programas de formação continuada que sejam capazes de propiciar uma sólida base teórica para subsidiar a prática dos agentes que atuam nas diversas instâncias da rede de proteção às crianças e adolescentes, sobretudo no que se refere ao desenvolvimento de uma cultura profissional comprometida com a ação coletiva.

Observou-se também as contradições que envolvem o enfrentamento da VSIJ, pois as principais dificuldades identificadas no enfrentamento da violência são também indicadas como as ações que apresentam maior potencial para a mudança da realidade, principalmente no que se refere ao trabalho em rede e à efetivação de políticas públicas direcionadas para esta questão.

Reiterando o argumento de que se trata aqui de um tema altamente complexo, torna-se imprescindível o desenvolvimento de estudos e pesquisas que contribuam para sua elucidação e instiguem as instâncias governamentais, os profissionais que atuam na rede e a sociedade civil a pensarem, seriamente, sobre o problema de pertinência abrangente, tendo em vista a intervenção coletiva no seu enfrentamento. Trata-se, no fundo, de admitir a importância e a urgência de encontrar caminhos para uma ação eficaz.

\section{REFERÊNCIAS}

1. Guimarães JATL, Villela WV. Características da violência física e sexual contra crianças e adolescentes atendidos no IML de Maceió, Alagoas, Brasil. Cad. Saúde Pública. 2011;27(8):1647-53.

2. Sanchez RN, Minayo MCS. Violência contra crianças e adolescentes: questão histórica, social e de saúde. In: Brasil. Violência faz mal à saúde. $1^{\text {a }}$ ed. Brasilia: Ministério da Saúde; 2006. p.29-38.

3. Gontijo DT, Alves HC, Paiva MHP, Guerra, RMR, Kappel VB. Violência e saúde: uma análise da produção científica publicada em periódicos nacionais entre 2003 e 2007. Physis. 2010;20(3):1017-54.

4. Paixão ACW, Deslandes SF. Análise das políticas públicas de enfrentamento da violência sexual infantojuvenil. Saude soc. 2010;19(1):114-26.

5. Giovanetti MAGC, Santos GL, Felizardo Jr LCF. Caderno do agente: enfrentamento à violência sexual infanto-juvenil nos Vales do Jequitinhonha, do Mucuri e região metropolitana de Belo Horizonte. Belo Horizonte: PROEXT UFMG; 2009.

6. Brasil. Aspectos jurídicos do atendimento às vítimas de violência sexual: perguntas e respostas para profissionais de saúde. $2^{\mathrm{a}}$ ed. Brasília: Ministério da Saúde; 2010.

7. Schwanck RH, Pauletti G, Zorzo JAT, Gomes VLO. A percepção de formandos de enfermagem acerca da violência contra a criança. Cogitare enferm. 2005;10(2):41-6.

8. Brasil. Plano Nacional de Enfrentamento da Violência Sexual Infanto-Juvenil. $3^{\text {a }}$ ed. Brasília: Ministério da Justiça; 2002.

9. Secretaria de Direitos Humanos. Programa de Ações Integradas e Referenciais de Enfrentamento da Violência Sexual Infanto-Juvenil no Território Brasileiro - PAIR. Brasília, DF: Ministério da Justiça; [s.d.]. [acesso em 04 set 2011]. Disponível: http://www.portal.mj.gov.br/ sedh/ct/pair-parametrosparadisseminação2007.pdf 
10. Bardin L. Análise de conteúdo. $4^{\mathrm{a}}$ ed. Lisboa: Edições 70; 2010.

11. Pinheiro RRLMP. A violência sexual infantojuvenil: em busca de uma avaliação. In: Programa de Pós-Graduação em Políticas Públicas. III Jornada Internacional de Políticas Públicas: Anais da III Jornada Internacional de Políticas Públicas; 2007; Universidade Federal do Maranhão. São Luis: Universidade Federal do Maranhão; 2007. p.1-7.

12. Costa MCO, Carvalho RC, Santana MAO, Silva LMS, Silva MR. Avaliação do Programa Nacional de Ações Integradas e Referenciais (PAIR) para o enfrentamento da violência sexual contra crianças e adolescentes, em Feira de Santana, Bahia. Ciênc. saúde colet. 2010;15(2):563-74.

13. Iwamoto HH, Oliveira RC, Camargo CF, Tavares LC, Oliveira LP. A violência sexual infanto-juvenil sob a ótica dos informantes-chave. Rev. Eletr. Enf. [Internet] 2010;12(4):647-54.

14. Brasil. Lei n. 8069, de 13 de julho de 1990. Dispõe sobre o Estatuto da Criança e do Adolescente. Brasília: Secretaria de Estado dos Direitos Humanos; 2009

15. Vargas JD, Silva KA. Diagnóstico de Uberaba, Teófilo Otoni e Itaobim: a rede de proteção. In: Cunha EP, Silva EM, Giovanetti MAGC. Enfrentamento à violência sexual infanto-juvenil: expansão do PAIR em Minas Gerais. Belo Horizonte: PROEXT UFMG; 2008. p. 154-80.

16. Ude W. Enfrentamento da violência sexual infantojuvenil e construção de redes sociais. In: Cunha EP, Moreira da Silva E, Giovanetti MAG. Enfrentamento à violência sexual infanto-juvenil-expansão do PAIR em Minas Gerais. Belo Horizonte: UFMG; 2008. p. 30-60.

17. Brasil. Guia Escolar: métodos para a identificação de sinais de abuso e exploração sexual de crianças e adolescentes. Brasília: Secretaria Especial dos Direitos Humanos e Ministério da Educação; 2004.

18. Gontijo DT, Medeiros M. Crianças e adolescentes em situação de rua: contribuições para a compreensão dos processos de vulnerabilidade e desfiliação social. Ciênc. saúde colet. 2009;14(2): 467-75. 